\title{
CONVERGENCE OF SEQUENCES OF DISTRIBUTIONS
}

\author{
R. M. DUDLEY
}

ABSTRACr. It is shown that in L. Schwartz's distribution space $D^{\prime}$, there exist sequentially open sets which are not open. The class of all sequentially open sets is not compatible with the vector space structure on $D^{\prime}$.

Let $(S, \Im)$ be a topological space. A set $A \subset S$ is called sequentially closed if $x \in A$ whenever there exist $x_{n} \in A, n=1,2, \cdots$, such that $x_{n} \rightarrow x$ for $J$. We call $(S, J)$ and $J$ sequential iff every sequentially closed set is closed.

T. Shirai [6, Theoreme 5] proved that L. Schwartz's space D of test functions, with usual topology, is not sequential. The main purpose of this note is to prove that the space $\mathfrak{D}^{\prime}$ of distributions also is not sequential.

We call a set sequentially open iff its complement is sequentially closed. A topological vector space $(S, J)$ is called convex-sequential iff the convex sequentially open sets form a base for $J$. It is easily shown that every bornologic locally convex space is convex-sequential [1, Theorem 6.3]. Thus all the spaces of the theory of distributions are convex-sequential with strong topologies.

Weak topologies are not usually convex-sequential. In distribution theory, weak convergence of sequences coincides with strong convergence. Then since the strong topologies are convex-sequential, the weak topologies must be neither convex-sequential nor sequential.

Let $C$ be a relation between sequences $\left\{x_{n}\right\}$ and points $x$ in a space $S$, written $x_{n} \rightarrow_{C} x$. If $F \subset S$ we say $F$ is $C$-closed iff $x_{n} \in F$ and $x_{n} \rightarrow_{C} x$ imply $x \in F$. The complements of $C$-closed sets form a topology $T(C)$. For any topology $T$, we have the relation $C(T)$ : convergence of sequences for T. J. Kisyński [3] proved that if $(S, C)$ is an " $L^{*}$. space," then $C(T(C))=C$. Earlier, F. Hausdorff [2] had come very close to the same result. Clearly $T(C(T))=T$ iff $T$ is sequential.

Unfortunately, the map $C \rightarrow T(C)$ from $L^{*}$-convergences into sequential topologies fails to have good functorial properties. If $\left(S_{1}, C_{1}\right)$ and $\left(S_{2}, C_{2}\right)$ are two $L^{*}$-spaces, we have a natural $L^{*}$-convergence $C_{1} \times C_{2}$ in the Cartesian product $S_{1} \times S_{2}$, but $T\left(C_{1} \times C_{2}\right)$

Received by the editors March 17, 1969 and, in revised form, April 2, 1970.

AMS 1970 subject classifications. Primary 46F05.

Key words and phrases. Sequential topology, sequentially closed set, topological vector space, Schwartz distributions. 
may strictly include the product topology $T\left(C_{1}\right) \times T\left(C_{2}\right)$ ! This possibility was apparently first discovered by J. Novák [4]. At the end of this note we will give some details of Novak's example, since it is simple and his original paper may not be easily accessible. In $[1, \S 9]$ I showed that $T\left(C_{1}\right) \times T\left(C_{2}\right) \neq T\left(C_{1}\right) \times T\left(C_{2}\right)$ for $D$ and $D^{\prime}$ with their usual convergences.

This difficulty with Cartesian products does not occur for convex open sets [1, Theorem 6.2]. But there is trouble in relativizing to subspaces even with convexity. A linear form on a convex-sequential vector space $S$ is continuous iff it is sequentially continuous. But a linear subspace $T$ of $S$ may not be convex-sequential. W. Słowikowski [7] has found, in certain cases, good conditions on $T$ which allow every sequentially continuous linear form on $T$ to be extended to a continuous linear form on $S$.

For still further examples of bad behavior of the map $C \rightarrow T(C)$, see $[1, \S 3]$. Although convex-sequential topologies have some of the same pathology as sequential topologies, convexity at least improves the situation somewhat.

But there are natural nonlinear maps which are sequentially continuous and not continuous. An example is the bilinear form $(T, f)$ $\rightarrow T(f)$ on $\mathfrak{D}^{\prime} \times \mathfrak{D}$. Thus it may be worth knowing which topologies are sequential as well as which are convex-sequential.

In $[1, \S 9]$, I recklessly made some statements about the sequential properties of distribution spaces, not founded on the results proved earlier in the paper or elsewhere. ("Theorems" 8.3 and 8.4 of [1] are false $[1$, correction $]$ but they are not at issue here.) I wrote that the strong topologies of $\mathcal{S}^{\prime}$ (the tempered distributions) and $\mathcal{E}^{\prime}$ (the distributions with compact support) are not sequential, but they are (J. H. Webb [9]). Actually, my Theorem 8.5 in [1] yields another proof that $\delta^{\prime}$ and $\mathcal{E}^{\prime}$ are sequential.

I implied at the end of [1] that $\mathfrak{D}^{\prime}$ is not sequential. This also was not based on any proof, but it happens to be true. Here (for the first time, to my knowledge) is a proof.

Proposition 1. $D^{\prime}$ is not sequential (for its usual strong topology).

Proof. We do the proof for the real line $R$; general noncompact domains can be treated similarly.

Let $\left\{\varphi_{r}\right\}_{r=1}^{\infty}$ be a countable dense set in $D(R)$. Let $\left\{U_{n}\right\}_{n=1}^{\infty}$ be a base of balanced convex open neighborhoods of 0 for the Fréchet space $D_{[0,1]}\left[5\right.$, p. 24], with $U_{n+1} \subset U_{n} / 2, n=1,2, \cdots$, and $\sup |f|$ $\leqq 1$ for all $f \in U_{1}$.

We define as usual the polar $A^{0}$ of a set $A \subset D$ : 


$$
A^{0}=\left\{T \in D^{\prime}:|T(f)| \leqq 1 \text { for all } f \in A\right\} .
$$

For any positive integers $m$ and $n$ let $f_{n m}$ be a continuous real function with support in $[0,1], \int f_{n m} \bar{\varphi}_{r}=0, r=1, \cdots, m$ (all integrals are for Lebesgue measure on $R$ ), and $\left[f_{n m}\right] \in U_{n+1}^{0} \sim U_{n}^{0}$. (Here $[f] \in D^{\prime}$ is defined by $[f](\varphi)=\int f \varphi, \varphi \in D$.) Note that a continuous $f$ with nonempty support included in $[0,1]$, orthogonal to $\varphi_{1}, \cdots, \varphi_{m}$, exists. $\lambda f \in U_{n+1}^{0}$ if and only if $|\lambda| \leqq \alpha$ for some $\alpha, 0<\alpha<\infty$, and then $\lambda f \notin U_{n}^{0}$ if $|\lambda|>\alpha / 2$.

Let $g_{n m}(x) \equiv f_{m n}(x-n)$ (note reversal of indices). Let $T_{n m}=\left[f_{n m}\right]$ $+\left[g_{n m}\right]$. Then the set $A$ of all $\left\{T_{n m}\right\}_{m, n=1}^{\infty}$ has no convergent sequences in $D^{\prime}$ except the eventually constant ones (a convergent sequence must be equicontinuous on $D_{[0,1]}$, hence $n$ must remain bounded, but then so also must $m$ ). Thus $A$ is sequentially closed.

Given any neighborhood $U$ of 0 in $D^{\prime}$, take a neighborhood $V$ with $V+V \subset U$. Then $\left[g_{n m}\right] \in V$ for $n$ large enough; and, for fixed $n$, $\left[f_{n m}\right] \in V$ for $m$ large enough. Hence $T_{n m} \in U$ for some $n$ and $m$, but $0 \notin A$ so $A$ is not closed. Q.E.D.

Note that a sequentially open set $W$ in $D^{\prime}$ with $0 \in W$ has many of the properties of a neighborhood of 0 : its polar is bounded, and it contains all distributions which vanish on a sufficiently large interval $[-n, n]$. If $\mathscr{D}_{m}^{\prime}$ denotes the space of distributions with support in $[-m, m]$, then $W$ includes a neighborhood of 0 in $D_{m}^{\prime}$ for all $m$. If $W$ is $D^{\prime} \sim A$ in the above proof, there is no sequentially open $V \subset D^{\prime}$ with $0 \in V, V+V \subset W$. Hence:

PROPOSITION 2. If $J$ is the class of all sequentially open sets in $D^{\prime}$, then $\left(D^{\prime}, J\right)$ is not a topological vector space.

On the other hand $J$ is a topology with the same convergent sequences as the usual topology. Addition and multiplication by scalars are jointly sequentially continuous. (In a related case, despite [8], the space of Mikusiński operators apparently has not been provided with a natural vector space topology.)

The proof of Proposition 1 above shows equally that the space $\mathfrak{e}^{\prime}$ of Radon measures, as strong dual of the continuous functions with compact support [5, pp. 15-17], is not sequential, and likewise for various intermediate spaces $D^{\prime m}$.

Here is Novák's example [4] on Cartesian products. Let $S_{1}=S_{2}$ $=$ rational numbers, $C_{1}=$ usual convergence. We define an $L$-convergence $C$ on $S_{2}$ :

$x_{n} \rightarrow_{C} x \neq 0$ if and only if $x_{n} \rightarrow_{C_{1}} x$;

$x_{n} \rightarrow_{C} 0$ if and only if either 
(a) $x_{n}=0$ for $n$ large enough, or

(b) $x_{n} \rightarrow \pi / m$ (usual convergence) for some positive integer $m$.

Let $C_{2}=C(T(C))$, an $L^{*}$-convergence by [3]. Let $D=$ the diagonal $\left\{(x, x): x \in S_{1}\right\}, E=D \sim(0,0)$. Then $E$ is $T\left(C_{1} \times C_{2}\right)$ closed, but its $T\left(C_{1}\right) \times T\left(C_{2}\right)$ closure is $D$.

\section{REFERENCES}

1. R. M. Dudley, On sequential convergence, Trans. Amer. Math. Soc. 112 (1964), 483-507; correction, ibid. 148 (1970). MR 30 \#5266.

2. F. Hausdorff, Gestufte Räume, Fund. Math. 25 (1935), 486-502.

3. J. Kisyáski, Convergence du type $\mathcal{L}$, Colloq. Math. 7 (1959/60), 205-211. MR 23 \#A615.

4. J. Novák, Sur les espaces (£) et sur les produits Cartésiens (\&), Publ. Fac. Sci. Univ. Masaryk No. $27 \dot{3}$ (1939). MR 1, 221.

5. Laurent, Schwartz, Théorie des distributions, 2nd ed., Publ. Inst. Math. Univ. Strasbourg, nos. 9, 10, Hermann, Paris, 1966. MR 35 \#730.

6. T. Shirai, Sur les topologies des espaces de L. Schwartz, Proc. Japan Acad. 35 (1959), 31-36. MR 21 \#4352.

7. W. Slowikowski, Extensions of sequentially continuous linear functionals in inductive sequences of (F)-spaces, Studia Math. 26 (1966), 193-221. MR 33 \#548.

8. R. A. Struble, $A$ genuine topology for the field of Mikusinski operators, Canad. Math. Bull. 11 (1968), 297-299. MR 38 \#1523.

9. J. H. Webb, Sequential convergence in locally convex spaces, Proc. Cambridge Philos. Soc. 64 (1968), 341-364. MR 36 \#5652.

Massachusetts Institute of Technology, Cambridge, Massachusetrs 02139 